Putting the nonsocial into social neuroscience: A role for domain-general priority maps during social interactions

Ramsey, Richard; Ward, Robert

\title{
Perspectives on Psychological Science
}

DOI:

https://doi.org/10.1177\%2F1745691620904972

Published: 01/07/2020

Peer reviewed version

Cyswllt i'r cyhoeddiad / Link to publication

Dyfyniad o'r fersiwn a gyhoeddwyd / Citation for published version (APA):

Ramsey, R., \& Ward, R. (2020). Putting the nonsocial into social neuroscience: A role for domain-general priority maps during social interactions. Perspectives on Psychological Science, 15(4), 1076-1094. https://doi.org/10.1177\%2F1745691620904972

\footnotetext{
Hawliau Cyffredinol / General rights

Copyright and moral rights for the publications made accessible in the public portal are retained by the authors and/or other copyright owners and it is a condition of accessing publications that users recognise and abide by the legal requirements associated with these rights.

- Users may download and print one copy of any publication from the public portal for the purpose of private study or research.

- You may not further distribute the material or use it for any profit-making activity or commercial gain

- You may freely distribute the URL identifying the publication in the public portal ?
}

Take down policy

If you believe that this document breaches copyright please contact us providing details, and we will remove access to the work immediately and investigate your claim. 
Journal:

Title: Putting the non-social into social neuroscience: A role for domain-general priority maps during social interactions

\section{Richard Ramsey ${ }^{1,2}$ and Rob Ward ${ }^{1}$}

${ }^{1}$ Wales Institute for Cognitive Neuroscience, School of Psychology, Bangor University, Bangor, Gwynedd, Wales, LL57 2AS, United Kingdom

2 Department of Psychology, Macquarie University, Sydney, NSW 2109, Australia.

Correspondence: r.ramsey@bangor.ac.uk/richard.ramsey@mq.edu.au

Key words: social neuroscience; social cognition; person perception; domain-specificity; priority maps; biased competition. 


\begin{abstract}
Whether on a first-date or during a team briefing at work, our daily lives are inundated with social information and in recent years research has begun studying the neural mechanisms that support social information processing. We argue that the focus of social neuroscience research to date has been skewed towards specialised processes at the expense of general processing mechanisms with a consequence that unrealistic expectations have been set for what specialised processes alone can achieve. We propose that for social neuroscience to develop into a more mature research programme, it needs to embrace hybrid models that integrate specialised person representations with domain-general solutions, such as prioritisation and selection, which operate across all classes of information (both social and non-social). To illustrate our central arguments, we first describe then evaluate a hybrid model of information processing during social interactions that: 1) generates novel and falsifiable predictions compared to existing models; 2 ) is predicated on a wealth of neurobiological evidence spanning many decades, methods and species; 3 ) requires a superior standard of evidence to substantiate domain-specific mechanisms of social behaviour, and; 4) transforms expectations of what types of neural mechanisms may contribute to social information processing in both typical and atypical populations.
\end{abstract}




\section{Introduction}

Human social interactions tend to occur easily, a fact that disguises the complexity of the mental processes involved. Attempts to understand the biological bases of human social interaction have been hampered by a relative lack of neuroscience research. Indeed, the history of human neuroscience has been dominated by the study of domain-general mechanisms that apply to all contexts, with the study of systems supporting social interactions left relatively neglected or ignored completely. Over the last 25 years, however, the cognitive and brain mechanisms that underpin social interactions have begun to receive considerable attention (Adolphs, 2009; Cacioppo \& Berntson, 1992; Frith \& Frith, 2012; Lieberman, 2007). An initial picture of social information processing has emerged that spans perceptual, cognitive, emotional and regulatory functions and their associated neural substrates, which has informed both basic research in psychology and neuroscience, as well as research in clinical and applied settings (Adolphs, 2010a).

A central thrust of social neuroscience research has been to delineate processes that are specific to social interactions, so-called domain-specific processes (i.e., processes that are tailored to particular stimulus or task features; Barrett, 2012). This makes sense as a starting point for any emerging field of research because it is important to differentiate the research programme from prior work, which in this case, had primarily focussed on domaingeneral processes (i.e., processes that operate across different stimulus or task features; Barrett, 2012). It is clear that valuable insight has been generated through this general approach (Adolphs, 2010a; Frith \& Frith, 2010; Kanwisher, 2010). At the same time, we argue that the focus on specialised mechanisms has been over-extended, and a correction is needed at this point for social neuroscience to develop into a more mature research programme. Such a developmental shift for the field holds the potential to drive substantive theoretical and empirical advances in the domain of social as well as cognitive neuroscience.

We argue here that an overreliance on specialised, domain-specific explanations has produced a misleading characterisation of the mechanisms involved in supporting social interactions, and skewed the focus of the literature onto relatively specialised processes at the expense of general processing mechanisms. Indeed, several recent suggestions have argued that mechanisms underpinning social information processing are likely to be a combination of domain-general and domain-specific processes, as well as the links between the two types of information processing (Barrett, 2012; Binney \& Ramsey, 2019; Ramsey, 
2018b; Spunt \& Adolphs, 2017; van Elk, van Schie, \& Bekkering, 2014). In other words, domain-general and domain-specific systems may play complementary roles in social cognition (Michael \& D'Ausilio, 2015), and understanding how these systems interact is likely to be a challenging but ultimately revealing line of future research.

As we unpack in the subsequent sections of the paper, a relatively narrow focus on domain-specific systems has led to a situation where researchers interested in social information processing expect too much explanatory power from the operation of domainspecific systems alone, and the role of domain-general processes in social interactions has been neglected. Further, our understanding of domain-general processes reflects the outputs of mature research programmes. For instance, domain-general processes associated with orienting of attention and sequencing of complex behaviour have been studied in depth, across thousands of studies employing a diverse set of methodological approaches and spanning multiple species, which has produced a substantial body of supporting evidence (Corbetta, Patel, \& Shulman, 2008; Duncan, 2010; Petersen \& Posner, 2012). Consequently, rather than largely ignoring or downplaying the role of domaingeneral processes, we suggest that there is considerable value in generating and testing alternative models of cognitive and brain function where the contribution of domaingeneral systems to social information processing is explicitly modelled.

In the current paper, therefore, we emphasise the relatively untapped value of hybrid accounts of social information processing, which place greater parity on domaingeneral as well as domain-specific processes. As we outline below, there are substantive implications for taking such an approach in terms of understanding the cognitive and brain mechanisms that underpin social behaviour. Indeed, as we describe, these models generate novel predictions compared to existing models of social information processing, and they are simpler in the sense that that they rely on fewer specialised components and processes. In other words, we argue that there exist ready-made solutions to questions surrounding social information processing, which take a domain-general form, and they should be investigated alongside specialised processing components.

To illustrate the key advantages of hybrid accounts of cognitive and brain function, we outline an example hybrid model of information processing during social interactions. Importantly, however, this is not to suggest that there is one hybrid account of social information processing; rather, a productive research programme is likely to generate many 
different hybrid accounts. By outlining one hybrid model, we aim to use it as a vehicle to highlight the value of hybrid accounts more generally, as well as to link it in with other recent hybrid accounts of mental function (Amodio, 2019; Binney \& Ramsey, 2019; Cabeza \& Moscovitch, 2013; Jefferies, 2013; Lambon Ralph, Jefferies, Patterson, \& Rogers, 2017; Ramsey, 2018b; Spunt \& Adolphs, 2017). Hybrid accounts make it clear that a higher bar of attainment needs to be set for what counts as convincing evidence for domain-specific explanations of social behaviour. Therefore, hybrid models provide an important call to action for those interested in social information processing by requiring a deeper evaluation of the evidence used to support domain-specific information processing claims.

Our example hybrid model integrates two independent lines of research that, to date, have seen little cross-talk between them. The first is work on person perception in social and cognitive neuroscience, which has primarily focussed on domain-specific systems (Adolphs, 2010a; Frith \& Frith, 2010; Kanwisher, 2010). Person perception is used in a broad sense here, which relates to sensory, cognitive and affective processes that are tied to social interactions. The second line of research is based on domain-general processes associated with orienting of attention, which involves selecting between competing stimuli in the environment as well as competing internal states, such as task goals (Corbetta et al., 2008; Duncan, 2010; Peterson \& Posner, 2012). More specifically, a large and growing literature has established the concept of priority maps. Identified in a neural circuit spanning dorsal frontoparietal cortex, priority maps integrate bottom-up cues to stimulus salience, with topdown behavioural relevance regarding such things as task goals, in order to guide subsequent behaviour (Bisley \& Goldberg, 2003, 2010; Fecteau \& Munoz, 2006; Ptak, 2012; Serences \& Yantis, 2006).

The two systems of representation and control have distinct attributes. The person representation system is largely modality and content specific. In contrast, the priority mapping system is largely generalised in its function. Nonetheless, a crucial third piece of the proposed model is that these two systems are reciprocally connected. Indeed, social signals from the environment, as well as stored person representations and current goals are in constant flux and exchange, which is partly mediated by integration across multiple distributed circuits. Therefore, much like models of biased competition that were outlined over two decades ago (Desimone \& Duncan, 1995; Duncan, Humphreys, \& Ward, 1997), a 
core feature of the model is a continually updating level of functional integration and interaction between signals associated with person representations and priority maps.

In the following sections, we first outline the basic structure of the proposed model, before evaluating it in a range of ways. First, we compare it to alternative models that make opposing claims, such as that control processes rely on specialised systems (e.g., Brass, Ruby, \& Spengler, 2009). We also compare it to other models that pay limited attention to domain-general processes (e.g., Lieberman, 2007). In both cases, we show how an emphasis on domain-general processing can result in important new predictions. Another important feature of this approach is that it leads to a cumulative science, relying on well-studied, and well-established domain-general cognitive and brain systems, and we ask how far these general systems can take us in our understanding of a specific domain. We go on to consider other implications of the model by showing how it can reveal substantial new insight into the basic systems that support social information processing, as well as clinical conditions whose pathologies are typified by disruption to social information processing. Finally, we place constraints on the generality of the findings proposed, in order to explain when and where the model is and is not relevant, before considering the merits and weaknesses of the model as well as ways that it can take the field forward in novel directions.

\section{A hybrid account of information processing during social interactions}

The proposed model of social information processing is a hybrid account in the sense that it relies in equal measure on domain-specific mechanisms that are tied specifically to social information processing, as well as domain-general processes. The basic structure of the model is based on three primary sources. The first source is Jerry Fodor's conception of mind, which distinguished between a set of specialised input modules and a central processor $^{1}$ (Fodor, 1983). The second source is work in the semantic cognition literature, which has demonstrated that the extraction of meaning from the environment is based on two primary systems of representation and control, which rely on distinct cognitive and neural architectures (Binney \& Ramsey, 2019; Lambon Ralph et al., 2017; Jeffries, 2013). The third source is the overarching principle of brain organisation proposed in models of biased

\footnotetext{
${ }^{1}$ Here we only use the term "module" to refer to functionally related brain regions. We do not use it to refer to additional features that were initially proposed by Jerry Fodor to define information processing modules.
} 
competition (Desimone \& Duncan, 1995; Duncan et al., 1997). In the following section we outline the basic structure of the model and how it relates to these prior accounts of cognition and brain function.

\section{Overall structure of the model: representation, control and biased competition}

Similar to models developed in the semantic cognition literature, the current model comprises domain-specific social representations, and domain-general systems for computation of behavioural priorities and guidance of behaviour (Lambon Ralph et al., 2017; Jeffries, 2013; Figure 1A). The representational system supports the acquisition and long-term storage of information related to other people (Figure 1B, left panel). Domaingeneral systems make up what we refer to as "control" processes: that is, neural processes which guide and direct behaviour so that it is coherent and effective. Guidance of behaviour in this way is achieved through the impact of control processes on proximate mechanisms of perception and cognition. As such, the control mechanisms that we discuss below have the effect of coordinating a broad range of specialised processors for perception, cognition, affect, and memory, in order to produce effective responses to relevant objects and persons in the environment.

In our model, control processes comprise priority maps and a process of biased competition. To be absolutely clear, we are not claiming that these two control systems comprise the entirety of control systems in human brains and behaviours. We see these as powerful examples of well-studied potential control mechanisms. Furthermore, these two examples reflect an interesting distinction for us: illustrating both a localisable and a distributed process for control. Priority maps guide behaviour to spatial locations of high behavioural relevance and have been associated with neural activity in dorsolateral frontoparietal cortex (Figure 1B, right panel). Biased competition operates in ubiquitous fashion across the entire brain and therefore integrates neural activity within and between the neural networks associated with person representation and priority maps, in line with task- and context-specific constraints (Figure 1C). 
Figure 1. Neural networks for representation and control in semantic cognition (A), social cognition (B) and as a function of biased competition (C).

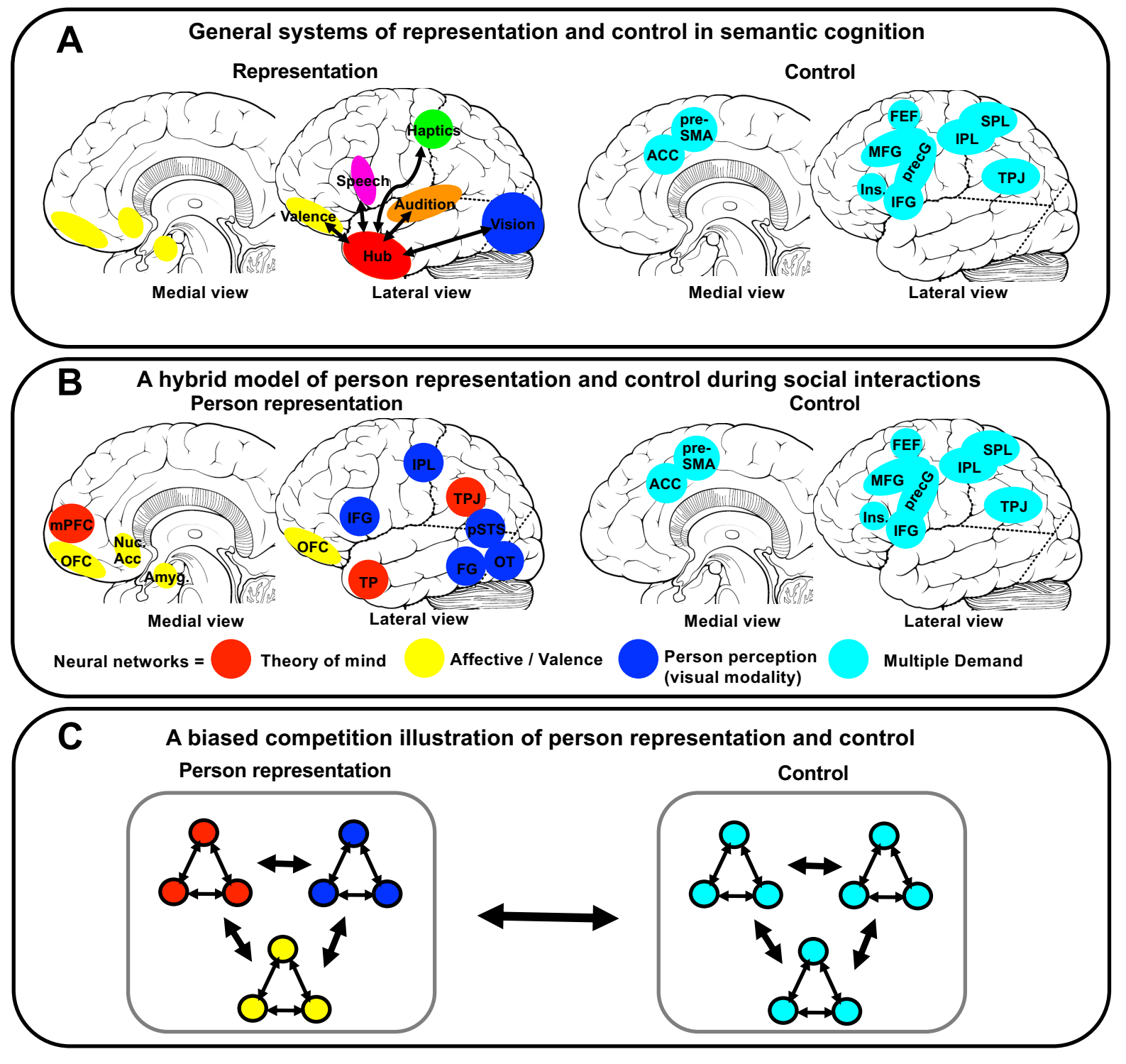

Figure 1. Illustrated in $(A)$ is the basic division between representation and control and the associated neural structures, which has been developed in the domain of semantic cognition (see Lambon Ralph et al., 2017; Jeffries, 2013). In (B) the division between representation and control is applied to mental processes that operate during ongoing social interactions. In (C) a model of biased competition is illustrated as it applies to representation and control. Under such a model, there is competition within and excitation between different forms of person representation (left panel), as well as different components of general cognitive control (right panel). Finally, there is excitation between person representations and control systems. Arrows denote competition and excitation within or between different information processing components.

Abbreviations $=\mathrm{OFC}=$ orbital frontal cortex; $\mathrm{mPFC}=$ medial prefrontal cortex; Nuc. Acc = nucleus accumbens; Amyg. = Amygdala; $\mathrm{ACC}=$ anterior cingulate cortex; $\mathrm{TP}=$ temporal pole $; \mathrm{IFG}=$ inferior frontal gyrus; $\mathrm{IPL}=$ inferior parietal lobule; TPJ = temporoparietal junction; $\mathrm{PSTS}=$ posterior superior temporal sulcus; $\mathrm{FG}=$ fusiform gyrus; OT = occipitotemporal cortex; pre-SMA = pre-supplementary motor area; MFG = middle frontal gyrus; precG = precentral gyrus; SPL = superior parietal lobule; FEF = frontal eye field; Ins. = insula. 
The model also shares some similarities with older conceptions of mind that suggested inputs are largely specialised, whereas central processes are largely generalised (Fodor, 1983). Under a broad conceptualisation of person perception, inputs for social cognition would include sensory, cognitive and affective person representations that span the perception of faces and bodies, as well as inferences about beliefs, traits, attitudes, valence and emotional responses. By contrast, central processes under this account, would be the control/prioritisation processes that take a generalised form, such that frontoparietal circuits regulate and ultimately prioritise responses based on interactions with signals from input nodes. We note that while both the representation system and priority maps might be usefully conceived as localised modules within the brain, the process of biased competition which integrates them, reflects highly distributed and non-localisable patterns of activity, that produce the crucial effect of allowing behaviour to be driven in a coherent way by a single object, event, or episode.

Representation in social information processing: cumulative person feature maps

Person perception in this context is broadly construed to reflect sensory, cognitive and affective processes. In short, person perception refers to any information that can cue who somebody is and how they are likely to behave. Therefore, such person representations comprise person feature maps across multiple levels of description, and they are cumulative in that they represent the current representational "state" based on the relevant history of social interactions with interaction partners. In the following, three broad sources of person information are outlined, which rely on largely distinct neural structures (Figure 1B, left panel).

The first system consists of largely perceptual processes within sensory systems, which so far have been investigated predominantly in the ventral visual stream (Kanwisher, 2010). Such circuits span fusiform and occipitotemporal cortices, which respond to depictions of faces (Kanwisher, McDermott, \& Chun, 1997) and bodies (Downing, Jiang, Shuman, \& Kanwisher, 2001; Downing \& Peelen, 2011), as well as responses to biological motion in posterior superior temporal sulcus (Grossman et al., 2000; Puce \& Perrett, 2003), and sensitivity to goal-directed actions in inferior frontal and parietal cortices (Caspers, Zilles, Laird, \& Eickhoff, 2010; Molenberghs, Cunnington, \& Mattingley, 2012). Other sensory 
modalities such as audition (Belin, Zatorre, Lafaille, Ahad, \& Pike, 2000), touch (Gazzola et al., 2012; Loken, Wessberg, Morrison, McGlone, \& Olausson, 2009; Morrison, Löken, \& Olausson, 2010), and olfaction (Insel \& Fernald, 2004), also contribute to the representation of others. The common feature amongst all these channels of information is that they permit the senses to detect distinct types of information about possible interaction partners, and they largely rely on functionally segregated neural circuits.

The second system of interest at the representational level is more cognitive in nature and relies on computations in the theory of mind network (C. D. Frith \& Frith, 1999). The theory of mind network spans anterior medical prefrontal cortex, temporoparietal junction and the temporal poles and is engaged across a wide variety of situations that involve reasoning about mental states, such as beliefs, desires and attitudes (C. D. Frith \& Frith, 1999; Saxe \& Kanwisher, 2003; Van Overwalle, 2009). Furthermore, the theory of mind network is engaged when making judgments regarding about another's traits or character (Mitchell, Cloutier, Banaji, \& Macrae, 2006; Mitchell, Heatherton, \& Macrae, 2002). Such inferential processes are largely different from the types of processing in sensory detectors, and they lead to the accumulation of a distinct, but complementary type of person knowledge. Such person knowledge representations regarding current beliefs and trait-based character are of course crucial for establishing how someone is likely to behave in a given social interaction.

The third representational system is associated valence and affect (Kuzmanovic, Jefferson, Bente, \& Vogeley, 2013; Vuilleumier \& Pourtois, 2007). Unlike the previous two representation levels, which may reflect processes that are largely tied to person perception and person inferences specifically, the processes associated with valence and affect that are likely to generalise to any situation, social or otherwise. In terms of neurobiological underpinnings during social interactions, the processing of valence and affect have been associated with a distributed circuit spanning the ventral striatum, amygdala, thalamus and cingulate cortex (Adolphs, 2010b; Insel, 2003; Kelley \& Berridge, 2002; Krach, Paulus, Bodden, \& Kircher, 2010; Pessoa \& Adolphs, 2010; Spunt \& Adolphs, 2019). In social contexts, individuals and groups will be tagged with a level of valence, which in part is likely to determine "liking", as well as approach and avoid behaviours (Insel, 2003; Kelley \& Berridge, 2002). As such, signals pertaining to valence are clearly important for forming 
impressions of others and guiding social behaviour, and make a distinct but commentary contribution to sensory and cognitive levels of representation.

Although these three processing streams rely on largely separate neural networks, it is likely that they also exchange signals through a process of functional integration (Park \& Friston, 2013; Sporns, 2013). That is, at some level, a coherent person representation requires integration across a range of person features (Greven, Downing, \& Ramsey, 2016; Greven \& Ramsey, 2017a, 2017b; Over \& Cook, 2018; Ramsey, 2018a). For example, one must be able identify physical features in order to identify who the other is in a social interaction, whilst also integrating and recalling other person knowledge, such as trait character judgments and valence (Over \& Cook, 2018; Ramsey, 2018a). Such integration may ultimately lead to more holistic person representations that abstract away from the specific features, as has been outlined for processing in anterior temporal cortices (Binney \& Ramsey, 2019; Lambon Ralph et al., 2017; Wang et al., 2017). Although of fundamental importance, much like functional integration research more generally (Park \& Friston, 2013), the neural systems that bind social information together is a relatively unexplored topic.

An important characteristic of these person feature maps is that they are cumulative, such that they represent the current representation of the person, given the historical accumulation of signals in those maps. That is, for each person feature map, the current representation is the result of the sum total of experience with that individual. Therefore, there is a memory component to person representations outlined in the current work (Amodio, 2019), which makes them cumulative. One implication is that the ongoing input during social interactions has to mesh and interface with stored person representation information.

\section{Control in social information processing: integrated priority maps}

We now turn to control processes, which in general have been argued to regulate, guide and manage other mental processes in order to shape human behaviour (Duncan, 2010; Peterson \& Posner, 2012). Priority maps have been studied extensively as a candidate system that guides the spatial focus of attention and ultimately regulates behavioural choices (Ptak, 2012). The basic premise follows the logic of stimulus salience maps, which suggested that the salient features of a stimulus drive the focus of attention (Itti \& Koch, 2001). As such, based on bottom-up signals from environmental cues, attention is directed 
towards the stimulus features that "win" the race to capture attention by virtue of being the most salient.

Of course, during social interactions, we frequently interact with others whilst maintaining a variety of goals. For example, whilst at a train station, we may be looking for a friend or trying to find the correct platform. As a consequence, the relative salience of other people versus platform information will be up- or down-regulated depending the individual's current goal. An important feature of priority maps, therefore, is that they are not restricted to the salience of stimulus features; instead, they have been shown to integrate stimulus features with current goal information (Bisley \& Goldberg, 2003; 2010; Ptak, 2012; Serences \& Yantis, 2006; Fecteau \& Munoz, 2006). Therefore, priority maps orient attention based on a qualitatively richer set of factors than stimulus features alone (Figure 2). Relatedly, more recent formulations of priority maps have expanded such factors to include a memory component (Awh et al., 2012), as well as motivational, affective and semantic processes (Todd \& Manaligod, 2018). In sum, priority in this broader sense reflects the perceived quality of the stimulus given relevant context and history, rather than an absolute physical quality of the stimulus (Figure 2; Ptak, 2012; Serences \& Yantis, 2006; Fecteau \& Munoz, 2006).

Although there are different flavours of priority map, they all have common foundational principles, which are the main focus of the current paper. First, priority maps have been identified in a dorsal frontoparietal network (Figure 1, right panels), which anatomically overlaps with the orienting systems outlined by others (Corbetta et al., 2008; Peterson \& Posner, 2012), as well as aspects of the multiple demand network that spans medial and lateral frontoparietal cortices (Duncan, 2010). Second, priority maps are feature, modality- and response-independent, such that they can take diverse feature information from different modalities and combine them into single coherent priority map, which then guides behavioural responses in a flexible manner that is not tied to a particular response effector (Ptak, 2012). Importantly for social interactions, which involve a multitude of signals, priority maps can integrate exogenous cues such as stimulus features with broader classes of signal that endogenous cues such as goals, memory, affective and motivational information (Figure 2; Awh et al., 2012; Todd \& Manaligod, 2018). As will become clear below, the domain-general functionality of priority maps is well-suited to orienting during 
social interactions, whilst at the same time making contrastive predictions in comparison to alternative models of social information processing.

Figure 2. Integration of endogenous and exogenous information during social interactions.

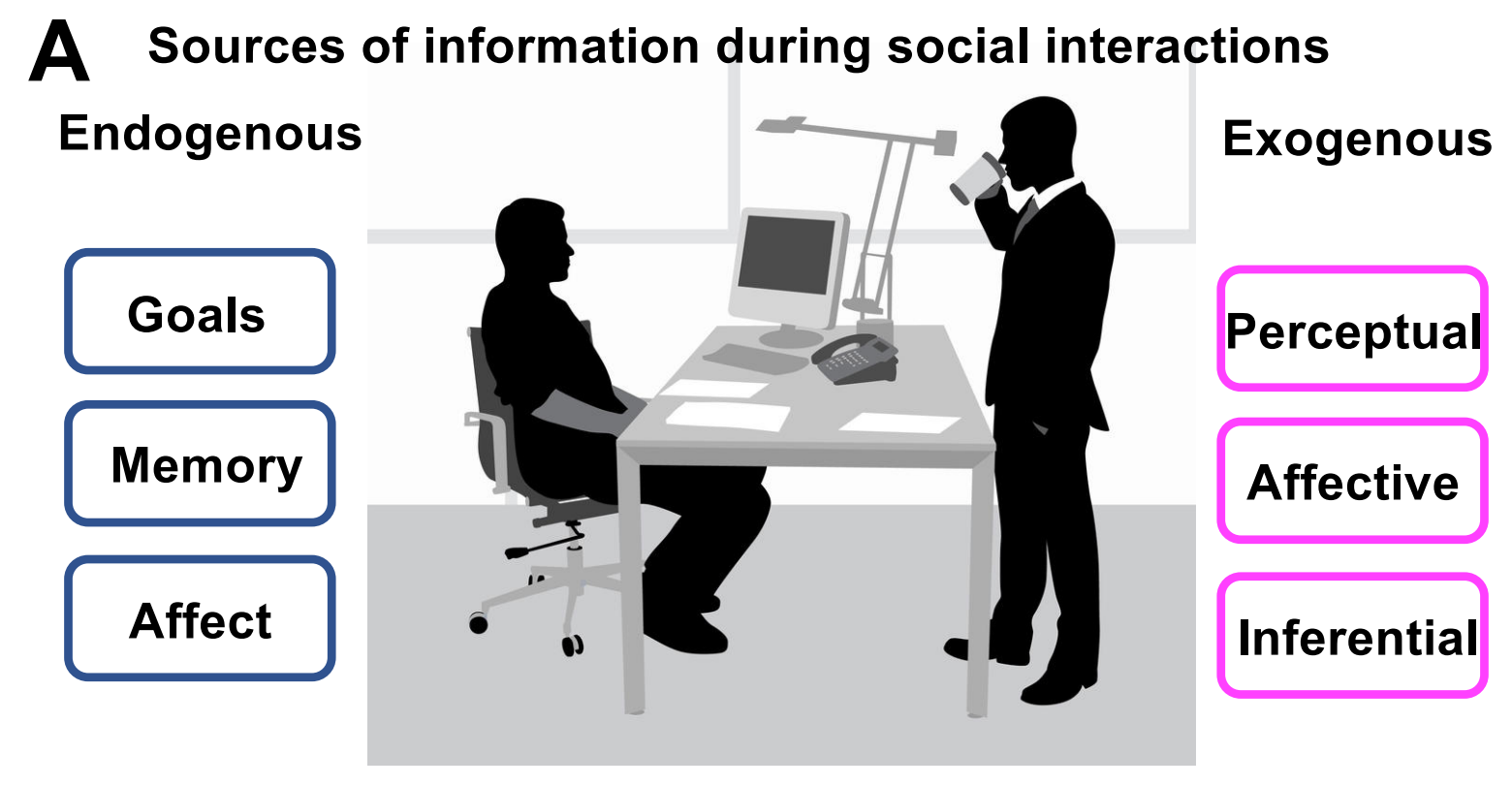

B

\section{Priority maps}
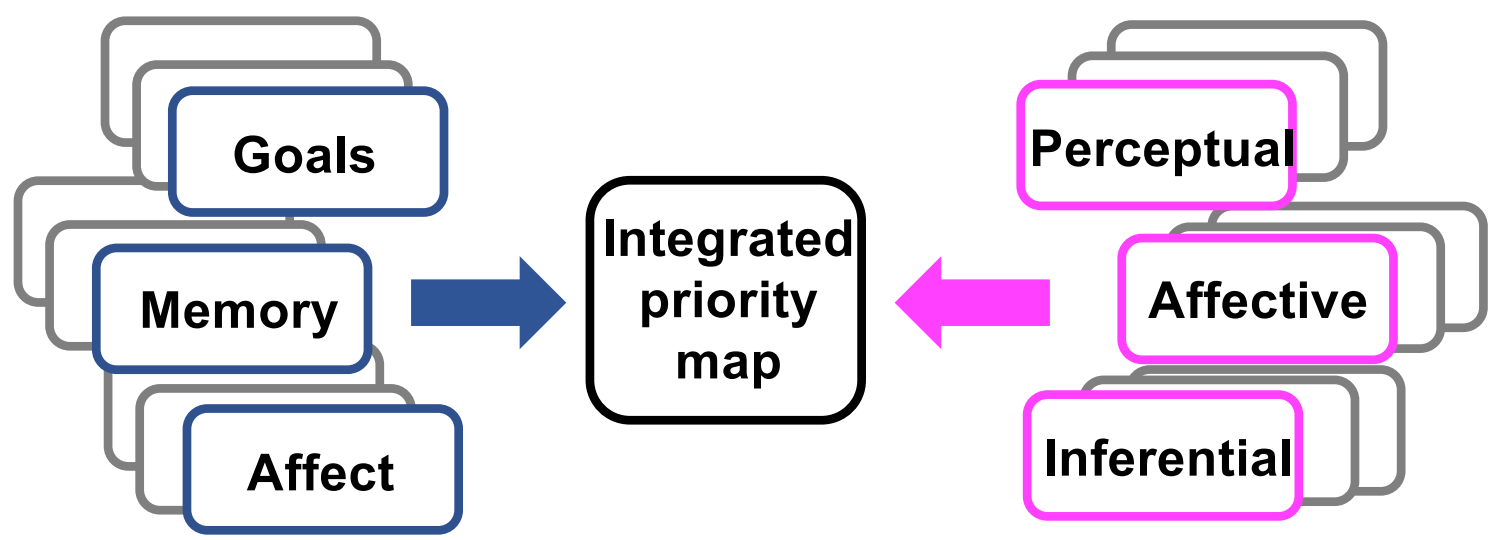

Figure 2. A) Two distinct sources of information need to be integrated during social interactions. These sources include endogenous cues, such as task goals, memory and affective states, as well as exogenous cues, such as perceptual, affective and inferential representations of other people. B) An integrated priority map is able to bring these two distinct sources of information together into a common space. By integrating information priority maps are able to incorporate diverse forms of bias, which ultimately guide the locus of attention. Shaded boxes reflect that endogenous and exogenous cues typically have a history and thus the current representation reflects one that emerges over time and not only reflects the current state. 
Integration within and between representation and control systems: biased competition

In principle, biased competition models offer a way to conceptualise how signals from different processing components may be integrated (Desimone \& Duncan, 1995; Duncan et al., 1997). Within a biased competition framework, the human brain can be characterised as a complex information processor that has many specialised processors operating in parallel. This would include specialised processors relevant for coding all kinds of objects, for example in the visual domain, form, motion, colour, as well as complex feature combinations, and regions with precise and invariant spatial coding. For coherent behaviour, the activity of these many different regions must be coordinated so that a single object or event guides response effectors. As we think beyond perceptual processing, we can see the need for coordinated brain activity that extends to specialised networks for memory, planning, and a range of executive functions.

Models of biased competition represent a general approach for achieving this coordinated brain activity (Desimone \& Duncan, 1995; Duncan et al., 1997). Biased competition, as achieved by competition within processors and excitation between, means that a bias for an object or event in one processor will tend to propagate through the network. Bias can arise from both stimulus factors (i.e., exogenous environmental salience), as well as from task goals or context (i.e., endogenous bias). In either case, the network converges onto the properties and the implications of a single object for behaviour. We can call this the "selected" object or event.

In our model, the systems of person representation and priority mapping, are functionally distinct and operate in parallel (see Figure 1). Biased competition addresses the serious danger in a system like this, which contains specialised, parallel processors: namely, behaviour can become incoherent if different processors are representing the properties of different objects. For example, what if the activity of the priority map was focused on one location, but the activity of the person representation was focused on a person at a different location? Since there is only one set of effectors, which of these systems would guide behaviour? Biased competition ensures coherent behaviour, as the properties of a single object or event will come to dominate across both principal systems (Figure 1C). Integration and coherent activity mean, for example, that activity from person representations can be integrated with current goals, history, affective and motivational factors constituting the current priority map. Within this context, we can see that brain 
regions associated with social stimulus processing, such as face- and body-selective brain areas, and also higher-level networks for theory of mind, are just other forms of specialised processors, and likewise require and benefit from integration and coordination with other brain activity. In fact, although not an explicit part of the model here, a process of biased competition could serve to integrate the variety of contextual, environmental, and task factors making up the priority map. This map would be updated in a dynamic fashion as social signals change over time.

There will be a diverse range of processors which might influence bias, as suggested in our discussion of person representation, and as illustrated in Figure 2, for example, processors for space, affect, goals, inferred states, and others. Biases from any and all processors might influence the final selected state, and conversely, none of them may be strictly required. In particular, we have been emphasising the role of spatial bias, in the form of a priority map. This is for good reasons, as social interactions involve other people, and other people occupy space. However, at any given time, the most influential sources of bias will vary according to context, and even space is just one (very useful) such source. For example, an internal affective state of happiness might bias competition so that a memory of a happy event comes to dominate processing. In this instance, the selected target of attention would be an internal representation, not an object present in the real world. However, in the context of an ongoing interaction with a nearby person, we would expect spatial bias to play a crucial role in guiding behaviour.

Our proposal is falsifiable. Several predictions follow from this proposed neurocognitive structure, and specifically, the idea that a biased competition, simultaneously operating over social representations and non-social or domain-general processes, would converge on a single object or event. First, and perhaps most importantly for the current argument, social stimuli gain control of behaviour through the same general mechanism as any other form of stimulus. There is no need for socially-specific forms of control. As long as there is a domain-specific representation of social stimuli, a general process can allow these representations to be selected and guide behaviour.

Second, social and non-social processors are expected to show mutual influence. Convergence and integration through biased competition means that both the social and non-social properties of the selected social object are selected concurrently. For example, an angry person occupies space, and so processing of this stimulus is subject to both social 
and spatial biases. A bias for angry stimuli may lead to convergence on an angry social object, with the result that all properties of this object, both social (e.g., identity, race, gender) and non-social (e.g., location, motion, form) are available to control behaviour.

A third and related prediction is that when an object has been selected, the final converged state of the network will be similar, regardless of the initial source of bias. For example, an angry person might be selected because of a bias towards anger, or because of a bias towards the location that person is occupying. In both cases, properties of the relevant person will be simultaneously activated and available to control behaviour.

Fourth, in the model, as the final converged state representing "selection" arises from biased competition over all processors, selection for social and non-social objects cannot be readily dissociated. That is, there will be no "social focus" of attention that is dissociable from a parallel "non-social" focus. Instead, there is a single focus representing the convergence of activity over all processors.

Finally, although our model has no domain-specific social control system, it can mimic deficits in social control. The structure of our hybrid model, with domain-specific person representation, and domain-general control processes, would suggest that specific deficits in the selection of social stimuli can be explained as deficits in the richness of representation on which competition operates, rather than a deficit in the general control process itself. For example, if the person representation system were degraded, then the processes of competition within this representation, and the excitation with relevant priority maps, would be less effective in driving behaviour (e.g., Ward, 1999). The network would converge more slowly and less reliably on the social object of interest. That is, even when domain-general control systems are intact, they will not be able to operate as effectively if the domain-specific representations they depend on are disordered.

\section{A comparison to alterative models and the data}

We use proposals put forward by Haig (2014) as the framework for comparing models, as well as how all these models compare against the accumulated data in systems neuroscience to date. For example, simplicity and explanatory breadth are emphasised as particularly important factors to consider when evaluating theories or models (Haig, 2014). We use this framework to compare the current model to two types of prior model, which 
each make contrasting predictions, as we feel comparison to multiple types of social information processing model can be instructive.

\section{A comparison to specialised versions of control}

Specialised accounts of control largely rely on the theory of mind network to control or regulate social interactions in some way. Such accounts are largely premised on control in terms of controlling self-other processes. For example, using a task that requires the inhibition of automatic imitation (Brass, Bekkering, Wohlschlager, \& Prinz, 2000), it has been argued that conflict between one's own action intention and another's action is regulated by the same theory of mind system that is engaged when representing other people's mental states such as beliefs, desires and attitudes (Brass et al., 2009; Sowden \& Shah, 2014; Spengler et al., 2009; Wang \& Hamilton, 2012). Therefore, such claims are founded on the idea that a neurocognitive system (the theory of mind network) that is domain-specific and dedicated to social information processing, is also involved in "regulating" social interactions between individuals via the control of self-other interactions. Of note for present purposes, domain-general forms of control, which we have outlined above, such as prioritisation, inhibition and selection, have not been explicitly mentioned in these accounts. As such, these accounts do not present roles for general and specific forms of control but instead only focus on the role of the theory of mind network and specialised forms of control.

On the one hand, during social interactions, it seems clear that there must be some system (or set of sub-systems) that distinguishes self from other, otherwise social exchanges would be incoherent (Decety \& Sommerville, 2003; Georgieff \& Jeannerod, 1998). On the other hand, however, there seems no a priori reason why such a process would necessarily need to rely on a specialised and domain-specific set of control processes. Indeed, as we illustrate in Figure 3, an information processing account of the inhibition of automatic imitation can be realised without the need for socially-specific forms of control (Figure 3). Further, the domain-general view has more explanatory breadth because it provides a plausible account of control processes such as priority mapping in all situations, those that include social interactions, as well as those that do not.

On this analysis, specialised accounts of any process (including control processes), run the risk of exploding in number to account for ever more social circumstances that may 
require different types of control process. Indeed, more and more supplementary systems of control may be required for a full account of social control mechanisms to emerge. A further complicating factor for specialised accounts of control is the added requirement for integration between specialised and generalised forms of control, and indeed, between different forms of specialised control. That is, although there are interesting and informative counter-examples following damage and disruption (Shallice \& Cipolotti, 2018), the mature human cognitive system tends to operate smoothly, whether interacting with others or making a cup of tea. Indeed, there is a coherent sense with which we work towards task goals in the presence of environmental cues. Therefore, any model of information processing during social interactions that rests partly on specialised control systems needs a solution to the combinatorial problem that occurs. This very problem has been repeatedly raised in robotic subsumption architectures, where action selection must be arbitrated among many specialised control systems (Brooks, 1991). Put differently, alternative accounts need to (a) explain how multiple systems of control, which include specialised and generalised forms, are integrated into a coherent action plan; and (b) given the need for integration with general control systems, detail the benefits that specialised control systems then offer. 
Figure 3. A model of information processing during the inhibition of automatic imitation (a social stimulus-response compatibility paradigm).

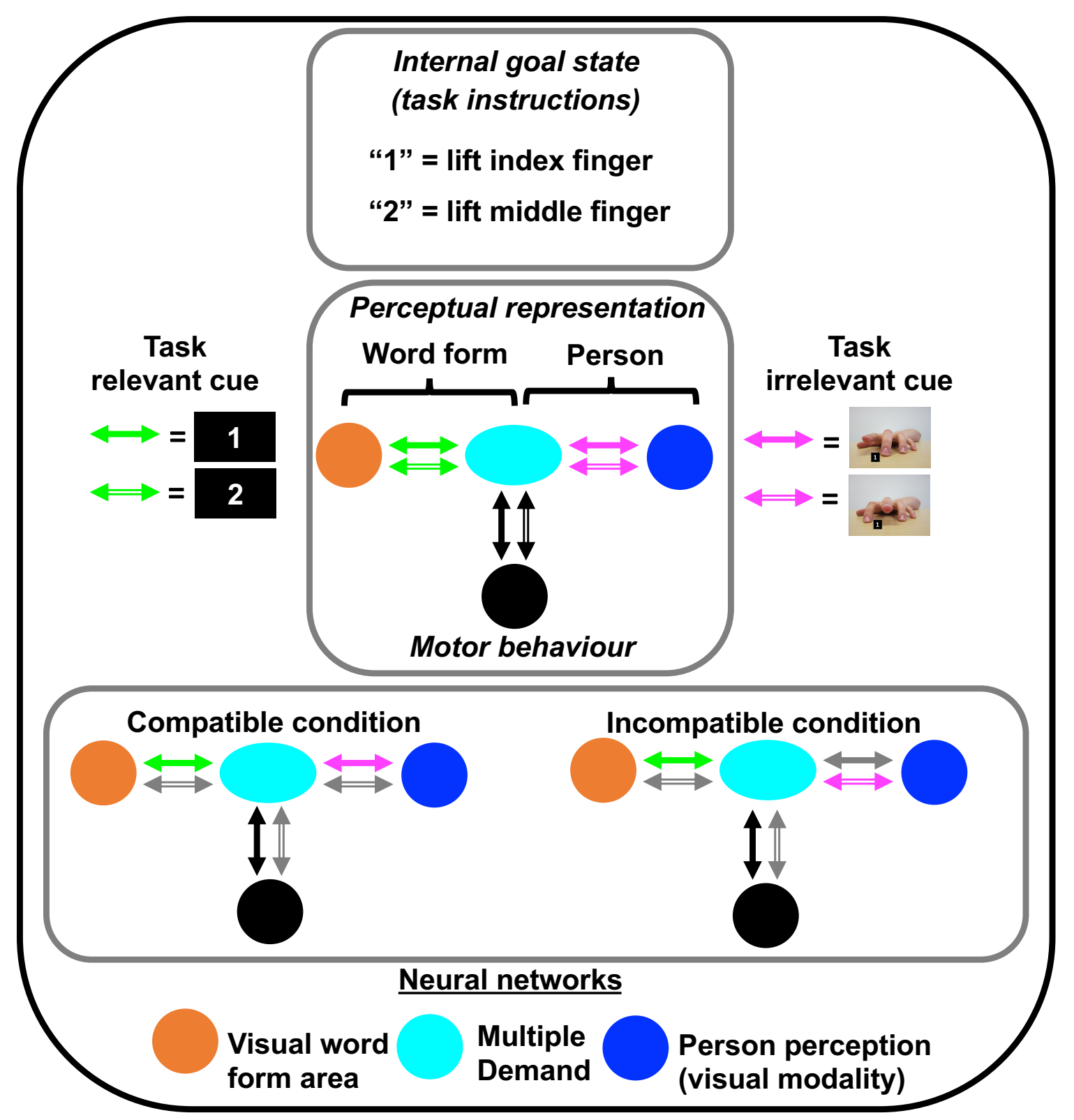

Figure 3. An alternative model of the processes involved in resolving a social stimulusresponse compatibility paradigm, that does not include any domain-specific forms of control. Instead, task-relevant cues are assigned a stimulus-response mapping, which is in relation to number cues and finger responses. Concurrently, there is also a task-irrelevant mapping that emerges, which is in relation to the observed finger movement and the associated motor response. Consequently, in the compatible condition, the task-relevant and task-irrelevant mappings both bias the same finger response. In contrast, in the incompatible condition the task-relevant and task-irrelevant cues, bias different finger responses and this conflict needs resolving before the correct response can be initiated. It is this conflict that leads to the reported reaction time difference between compatible and incompatible conditions. In contrast to Brass et al. (2009), we do not assume that the need to distinguish self from other generates conflict in this task nor do we require the engagement of the theory of mind network to resolve this kind of conflict, as under our 
formulation there is nothing special about this type of stimulus-response mapping conflict. Rather, it is just another form of stimulus-response mapping and as such general systems for prioritisation and control, which are coordinated through frontoparietal cortex, can engage with domain-specific word and person (finger) representations in more specialised representation systems.

\section{A comparison to general theories of social cognition}

General theories of social cognitive neuroscience have made a valuable contribution to the field by stipulating the range of processes that are likely to be involved in social information processing (Adolphs, 2009, 2010a; Lieberman, 2007; Frith \& Frith, 2010; 2012). These general theories frequently distinguish between social perception, cognition, and regulation (Adolphs, 2010a). Social perception and cognition would map directly onto the representational level of the current model, whereas social regulation would reflect the control component in the current model. Although cognitive control is mentioned briefly in several of these general accounts of social cognition as a supervisory or integration system (Adolphs, 2009; 2010a; Frith \& Frith, 2012), it receives minimal attention and lacks a detailed description on a neurobiological level. As such, general models of social cognition place little focus on generalised control processes, which leaves them relatively neglected.

When making comparison to our model, the first and most important thing to note is that there is no direct conflict between these general accounts and the current proposal. Rather, there is a difference in the emphasis and associated level of detail that is placed on generalised forms of control process. We propose a much larger role for generalised control processes, and attempt to demonstrate how they have direct consequence for how social information processing is conceived, how theories are generated and the types of hypotheses that might serve to be most fruitful in understanding social brain mechanisms. In contrast, these prior general accounts minimise the role of generalised control. As we demonstrate below in the implications section, although these theories may be qualitatively equivocal on grounds of cognitive architecture, we feel there are important reasons to increase the focus on generalised control processes as they have important knock-on consequences for how we may understand relationships between distinct processing components, and thus illuminate the structure of cognitive and brain systems more generally. 


\section{Compared against current empirical evidence}

In this subsection, we compare the central tenets of the model to the extant literature in social and cognitive neuroscience. We take each component of the model and compare it against the data in turn. First, we consider the representational level of the model. Although the exact nature of selectivity in the visual system, for example, is debated (Tarr \& Gauthier, 2000; Yovel \& Kanwisher, 2004), there is consensus in broad terms regarding functional segregation as a cardinal feature of brain organisation (Park \& Friston, 2013) and in particular in reference to social information processing (Kanwisher, 2010; Adolphs, 2009; 2010a). Therefore, at a broad level, the one that we are focussing on here, there is widespread agreement that largely distinct processing components show sensitivity to information that pertains to other people in terms of sensory and perceptual processing as well as mental state ascription. In sum, there is strong evidence for a set of distributed neural circuits that are preferentially tuned to process a range of person-related information (Kanwisher, 2010).

Second, as previously acknowledged, there is considerable evidence that details neural structures that support generalised forms of cognitive control. Indeed, the proposal that generalised forms of control are associated medial and lateral frontoparietal cortices, is supported by a comprehensive evidence base, which has sufficient depth and breadth to engender confidence in the claims being made. For instance, the evidence consists of thousands of studies in humans across a range of complementary methods, and is also supported by comparative work in non-human species (for reviews, see Badre, 2008; Corbetta et al., 2008; Desimone \& Duncan, 1995; Duncan, 2010; Miller, 2000; Petersen \& Posner, 2012). The further proposal that one component of these generalised systems of control concerns orienting of attention and priority mapping, and that this relies on dorsal frontoparietal cortex, is also supported by a wealth of evidence (Awh et al., 2012; Todd \& Manaligod, 2018; Bisley \& Goldberg, 2003; 2010; Ptak, 2012; Serences \& Yantis, 2006; Fecteau \& Munoz, 2006).

Third, and by contrast, there is relatively weak evidence for specialised forms of control. There is some evidence that specialised forms of control exist to regulate social interactions, which rely on the operation of the theory of mind network, but it is relatively 
weak in the sense that it relies on a small number of studies, which lack powerfully designed replications (Brass et al., 2009; Sowden \& Shah, 2014; Spengler et al., 2009; Wang \& Hamilton, 2012). One specific weakness of the current evidence is that some of the neural claims lack clear evidence of functional specificity, which means that such evidence could reflect the operation of more general processing systems. In addition, much of the evidence from $\mathrm{fMRI}$ relies on reverse inference, whereby activity in a given anatomical region is taken to be indicative of a functional signature due to a brain region's prior association with certain functions (Poldrack, 2006). The use of reverse inference is particularly important for regions like medial prefrontal cortex and temporoparietal junction as they have a particularly heterogenous functional profile at the systems level (Amodio \& Frith, 2006; Patel, Sestieri, \& Corbetta, 2019; Rushworth, Buckley, Behrens, Walton, \& Bannerman, 2007).

Take temporoparietal junction, for example, which has been consistently implicated in orienting of attention and social cognition (Patel et al., 2019). Without clear distinction between social and non-social processes, one could wrongly conclude that the region's performance in a given task is more specialised than it is. Moreover, if spatially distinct portions of temporoparietal junction show different effects that relate to social and attentional functions (Scholz, Triantafyllou, Whitfield-Gabrieli, Brown, \& Saxe, 2009), then it seems even more important to functionally distinguish between these subdivisions when making specific claims about this area (Krall et al., 2015). Further, the largest fMRI work to date that used a multi-experiment, high-power approach (Darda, Butler, \& Ramsey, 2018), as well as a meta-analysis of all related fMRI work (Darda \& Ramsey, 2019), shows very limited evidence for specialised control processes in the context of imitation inhibition, but compelling evidence for generalised control. Such evidence contrasts with the initial fMRI research on imitation inhibition that used much smaller sample sizes, and argued for a socially-specific form of control in right temporoparietal junction (Brass et al., 2001; 2003; 2009; Spengler et al., 2009). Therefore, the sum total of evidence for specialised control systems is weak. This said, a lack of evidence does not rule out the plausibility of such a system in principle. Rather, it means that the theory should be re-evaluated in light of this evidence, before further claims are made regarding specialised control systems during social interactions. 
Fourth, there is limited evidence to date that shows how systems of representation and control interact during social interactions. The term limited in this sense means that only a few studies have examined links between representation and control when compared to the work that has studied them separately. Some work shows domain-general interactions with person representations, either directly by functional integration measures (Baldauf \& Desimone, 2014; Quadflieg et al., 2011), or less directly by proposing distinct but complementary processing components for specialised and generalised systems (Ramsey, Hansen, Apperly, \& Samson, 2013). The latter approach follows a long tradition of studying theory of mind in cognitive psychology that proposes complementary roles for perspective computation and perspective selection, which reflects specialised and generalised processes, respectively (Leslie, German, \& Polizzi, 2005; Leslie \& Thaiss, 1992).

Other work suggests that domain-specific systems, such as the theory of mind network, provide a controlling signal that modulates person representations (Wang \& Hamilton, 2012; Wang, Ramsey, \& Hamilton, 2011). One piece of evidence for this claim comes from an fMRI study using functional connectivity analyses. Wang and colleagues (2011) showed that the response in medial prefrontal cortex influenced activity in frontal and temporal cortices when a person looks at you during an imitation inhibition task. Although it is plausible that medial prefrontal cortex provides a control signal over other person representations, the claim is currently based on a small number of studies, and it is hard to distinguish from an account based on functional integration across different types of person representation. For instance, an explanation at the person representation level would suggest that when someone looks at you it influences the intentions and mental states that you ascribe to them. In an imitative context, such mental state person information would need to be integrated with other person features, such as observed action representations, and this may occur through functional integration between neural networks. Therefore, an updated person representation that shares multiple features (perceptual and inferential) could also account for the same findings (Over \& Cook, 2018; Ramsey, 2018), without the need to engage specialised control processes, per se. Therefore, more work is needed, in terms of understanding integration between levels of representation and control. 


\section{Implications for understanding cognitive and brain mechanisms of social information processing}

By far the broadest implication of the current proposal is that a purely piecemeal approach to understanding any cognitive faculty (social or otherwise) is fundamentally limited (Churchland, 2013). Take attempts to understand vision, for example. Vision relies on general intelligence competencies that make one aware of concepts and guide expectations; vision is not reducible to mental operations housed within visual cortex alone, such as edge, colour, motion and shape sensitivity (Churchland, 2013). The systems that support social interactions are likely to be no different; they may just include a more varied set of signals and hence more integration and control. To be clear, a piecemeal approach is valuable in its own right and it has made a sizable impact in social neuroscience and it will continue to do so. At the same time, such an approach is limited in important ways, which we feel has been relatively neglected in social (and human) neuroscience and ultimately detrimental to progress.

Based on this first broad implication, we suggest that social neuroscience needs to change its default approach and embrace general processes alongside specialised processes from the outset. A deeper appreciation and acknowledgement of domain-general processes in social cognition means that non-social, but well-studied processes, can offer valuable insight into the cognitive and neural processes associated with navigating social interactions. A similar argument has been made for understanding memory (Amodio, 2018; Spunt \& Adolphs, 2017), semantic (Lambon Ralph et al., 2017; van Elk et al., 2014) and motor control systems (Cisek, 2007; Cisek \& Kalaska, 2010). Indeed, much like for these other areas of research, ignoring generalised processes seems equally inefficient, given their potential relevance to the problem at hand. Consequently, these more established research programmes can help guide expectations regarding the division of labour between more specialised and general systems.

Moreover, considering the substantial and multi-method evidence for the involvement of frontoparietal brain circuits in prioritisation, any specialised claims should show that the results do not reflect operations of the domain-general system. In other words, the burden of proof is on specialised accounts to present compelling and consistent evidence that specialised brain circuits and processes underpin social control. Moreover, 
domain-general processes, by definition, operate across all contexts (to some extent), which would therefore include social contexts. In terms of explanatory mechanisms, therefore, we may expect less from specialised control process, but more from generalised control processes. This suggestion is important because it is in direct opposition to the modal and dominant approach in social neuroscience.

A further broad implication of the current approach, which was previously outlined (Adolphs, 2009), is that it emphasises the importance of distinguishing between inputspecificity and mechanism-specificity. That is, just because a social process or behaviour may rely, in part, on a generalised control process, it does not prevent it from being a fundamentally social process or behaviour. Indeed, representational content, such as a human face or an emotional response, can be unambiguously social without the need for every mental process associated with it to be specialised and domain-specific. Although this has been made clear many times before, it is worth repeating as it has important consequences for what we can expect from social processes. Moreover, it is a central aspect of the current model that generalised control processes are the first calling point when modelling control processes during social interactions. In short, social cognition is still interesting if it relies partly on general processing mechanisms. That is, one can attempt to explain fully-fledged social processes with a combination of general and specialised mechanisms (Spunt and Adolphs, 2017), just as has been argued in the domain of memory (Cabeza \& Moscovitch, 2013).

The current proposal has straightforward clinical implications. If the basic cognitive and brain systems that operate in social contexts are less specialised than has been previously acknowledged, especially in terms of control and regulation of other processes, then it substantially changes the likelihood that an atypical domain-specific information processor may underpin the condition. In other words, the range of possible socially-specific mechanisms that may operate in an atypical manner may be a lot narrower than is typically considered. Likewise, any characterisation of a disorder of social information processing should also explicitly model general information processing components because the range of social difficulties need not be underpinned solely by specialised, socially-specific information processors. A related clinical implication is that deficits in the control of social processing can arise as a consequence of deficits or degradation in the systems for domainspecific social representation. For example, a difficulty in identifying emotional states in 
others could lead to inappropriate behaviour in a given the social context, which might otherwise look like a deficit in the control or regulation of social actions. Generally speaking, deficits in domain-specific representation can mimic a deficit in domain-specific control. In seeking to understand clinical social disorders, we must therefore look first to whether the social information is being encoded and accessed effectively.

\section{Constraints on generality}

Constraints on generality are important to identify because they place explicit limits on the scope of the claims being made, rather than letting implicit assumptions dictate how others may interpret a given proposal (Simons, Shoda, \& Lindsay, 2017). In the following, we outline three constraints on generality. First, the characterisation of social information processing presented above does not in principle preclude any form of domain-specific, social control. Rather, the model says that given an assessment of the theoretical underpinnings, as well as the evidence to date, it is simpler and has more explanatory breadth without the need for specialised forms of priority mapping in social contexts. Therefore, specialised forms of control are possible, but they are not necessary and they have limited empirical support to date.

What we are saying, however, is that the bar for identifying a domain-specific process of control should be high. We suggest that convincing evidence for domain-specific control systems would be based on the following principles, as borrowed from research on the visual system, which has identified tuning functions for particular classes of objects that are tied to distinct patches of visual cortex (Kanwisher, 2010, 2017). First, at a general level, evidence for a separate specific control system that applies to social interactions should be dissociable from social representations, as well as general forms of control. Second, the evidence should come from multiple methodological approaches (e.g., patients, neuroimaging, neurostimulation and comparative) and satisfy new recommendations from the open science movement regarding improvements in methodological rigour, such as replication, pre-registration and the use of powerful designs, among a host of other things (Munafò et al., 2017). More generally, claims regarding specificity of function would benefit from considering the principles recently put forward in another heavily studied domain, 
which relates to inferring emotions and facial expressions (Barrett, Adolphs, Marsella, Martinez, \& Pollak, 2019).

Second, we are modelling information processing here during ongoing social interactions. We are not covering the whole of social information processing or the many varied relationships between different types of general and social processes. Instead, our main focus in the current paper is that at any given point in time, one has a representation of the world (and people in it) along with a set of task goals. In other contexts, such as semantics, for example, there may well be general control processes, and more specialised control processes for social semantics, which engage ventral portions of frontoparietal cortex (see Binney \& Ramsey, 2019). We are also not covering the emergence and development of social representations i.e., representational content (Meyer, Bekkering, Haartsen, Stapel, \& Hunnius, 2015; Weigelt et al., 2014), or how expertise shapes such representations (Bukach, Gauthier, \& Tarr, 2006; Gerson, Bekkering, \& Hunnius, 2015). We speculate that learning about other people along with social concepts are also likely to involve integration between general control and input modules. But this is for research to pursue further. We also do not attempt to cover the entire range of possible relationships between different types of general and specifically social processes. Given that general processes operate across a diverse set of situations to some extent, we would expect the relationships between general and specifically social processes to be largely unrestricted. That is, we expect a range of general processes that relate to attention, memory, semantics and motor control to be relevant to a range of processes related to person perception and interaction that span perceptual, affective and inferential (i.e., theory of mind) mechanisms. Only following further research may we want to tighten these initially loose constraints.

Third, even though large sections of frontoparietal cortex are consistently engaged across a range of tasks (Duncan, 2010; Fedorenko, Duncan, \& Kanwisher, 2013), this does not preclude functional specification (by degree) in sub-sections of the network (Cole et al., 2013; Petersen \& Posner, 2012). The nature and structure of such fractionation in the multiple demand network is largely unknown and needs to be established. For example, it is possible that within the multiple demand network, there are dedicated sub-systems that are involved more (to some extent) with some more than other types of representational input. Relatedly, it is possible that control is similar in a qualitative sense, but it just operates on different inputs (i.e., social inputs), which makes the multiple demand network potentially 
graded in its operation. A graded structure would be one that is largely based on general processes, with some smaller proportion of sub-specialisation. However, this is still a very different model of control from the type of social control that is regularly offered in the social domain, which is that social networks associated with reward, empathy and theory of mind provide control in social contexts (Brass et al., 2009; Sowden \& Shah, 2014; Spengler et al., 2009; Wang \& Hamilton, 2012). Therefore, we are making a claim that control will predominantly take a general form and rely on the multiple demand network, with only a limited contribution from social circuits such as the theory of mind network. Hence, we are making a claim on a systems level about a grossly-defined neural network (multiple demand vs. specialised), which does not rule out functional division within a given network.

\section{Strengths and limitations}

A key strength of the proposed model is that it makes clear predictions, which can be falsified. If evidence accumulates to suggest that there are dedicated control mechanisms that are specific to social contexts, it would refute one basic tenet of the proposed model, which suggests that a largely domain-general priority mapping system interfaces with largely specialised input modules. For a convincing domain-specific claim to be made, however, such evidence would have to be based on the principles that we outlined in the previous section regarding the type and quality of evidence required. A call for clearly specified predictions is consistent with recent recommendations (Gray, 2017), which have emphasised the importance of being able to clearly articulate the features of a theory or model, in order for them to be tested. As such, model testability, as well as specifying constraints of generality, are of key importance to lay a foundation for a productive research programme, and we outline both of them here.

A possible criticism is that we present an overly simplistic account, which artificially reduces the complexity of social information processing. We would respond, however, that for a field that has emerged only very recently in the last 25 years, we may need to walk before we can run. That is, it may be time to reconsider that the complexity of the signal and associated mental computations that underpin social interactions does not necessitate a combinatorial explosion of processing systems to be articulated. Put differently, maybe as a field we need to take a moment to step back and re-evaluate some core assumptions, 
such as how much focus we should place on generalised systems (Amodio, 2018; Spunt \& Adolphs, 2017; Michael \& D'Ausilio, 2015), in order to make firmer progress. On the other hand, if this is an overly simplistic account, it will be easy to falsify. It would also be easy to point to robust evidence or theory that argues against the current formulation. Therefore, we think that the current formulation adds value to the field of social and cognitive neuroscience given that it has not focussed on the integration of domain-specific and domain-general processes, even though some have suggested that they could be complementary (Michael \& D’Ausilio, 2015).

\section{Open questions and future directions}

The preceding analysis raises more difficult questions than clear answers. We focus on two broad questions that we hope will stimulate distinctive future directions and result in significant research progress. The first question surrounds the extent to which control processes in social and non-social contexts are qualitatively and/or quantitatively different, and whether or not they rely on distinct information processing components. A quantitative difference would emerge if the same basic system of control, which is underpinned by the multiple demand network and resembles a domain-general priority map, is engaged to different degrees in social and non-social contexts. A qualitative difference would emerge if a different type of control is engaged during social interactions, which is distinct from a domain-general priority map, and possibly engages a different brain network. Based on consideration of this distinction, several questions follow for future research. Does this distinction help to set boundary conditions between social and non-social cognition? Is social cognition primarily different to non-social cognition in terms of the type of inputs or representational-level content, whist control processes are largely similar?

If priority maps link together saliency maps and behavioural relevance in a completely independent sense (i.e., a feature-, modality- and response-independent representation of the environment), can we add domain-independence to this list (social, non-social)? Are there just more diverse and complex types of input in the social domain, such as emotions, beliefs, desires, attitudes and trait/character judgments? Under this view, the mechanisms that control social representations are not special in any sense. Instead, other people are only special in one sense; they have some unique features in comparison 
to inanimate objects and other animals. But the same can be said of inanimate objects and other species - for example, planes can travel at 30000 feet above ground and birds can fly, but humans cannot (unaided). Therefore, the fact that humans have some unique qualities is not in itself unique (Adolphs, 2009). On this basis, we may need to temper expectations regarding the need for specialised control mechanisms during social interactions.

The second big question that this analysis brings to the forefront regards functional integration (Bullmore \& Sporns, 2009; Park \& Friston, 2013). Estimating, investigating and characterising the relationship between generalised control processes, such as priority maps, and person representations will be important for future work. Some work has already begun on this front, but more is needed, given that it is likely to be complex. Indeed, it may be the interaction between domain-general and social inputs that distinguishes social from non-social information processing streams. Also, what about integration between social modules, such as perceptual and inferential processes (Over \& Cook, 2018; Greven et al., 2016; Ramsey, 2018). How do these "holistic" representations interact with domain-general priority maps? This gets complex quickly, but that is all the more reason to learn from wider and more developed research programmes, such as semantic cognition (Lambon Ralph et al., 2017) and attention (Peterson \& Posner, 2012), as it may help researchers in social neuroscience specify more precisely what can already be expected from generalised control processes in terms of explanatory value.

\section{Conclusion}

For an emergent field of research, the initial job of carving out an existence has been sufficiently achieved by social neuroscience. Now, in order to develop into a more complete research programme, we argue that it cannot only attempt to understand one part of a system at a time; instead, it needs to consider component parts in the context of other constituent parts. As we have outlined in the current paper, for example, models of social information processing would benefit from explicitly incorporating the integration of specialised and generalised information processing mechanisms. Although this suggestion may sound obvious to some, the consequences of doing so are substantial. First, as we have demonstrated, it re-shapes expectations regarding the division of labour between specialised and generalised systems, which in many cases may substantially reduce the 
expected role of specialised mechanisms in social information processing. Second, if one considers the scarce and costly nature of scientific resources, it seems important to harness understanding gained from more established disciplines, such as domain-general cognition, and thereby avoid the danger of inadvertently reinventing the wheel of general cognition in a social guise. In summary, the general approach that this paper advocates and defends represents a substantial change to the modal approach in social neuroscience and would therefore have consequences for basic theory development as well as clinical disorders whose symptoms are typified by impaired social information processing. 


\section{References}

Adolphs, R. (2009). The Social Brain: Neural Basis of Social Knowledge. Annual Review of Psychology, 60(1), 693-716. doi:doi:10.1146/annurev.psych.60.110707.163514

Adolphs, R. (2010a). Conceptual Challenges and Directions for Social Neuroscience. 65(6), 752-767. Retrieved from http://linkinghub.elsevier.com/retrieve/pii/S0896627310001777

Adolphs, R. (2010b). What does the amygdala contribute to social cognition? Year in Cognitive Neuroscience 2010, 1191, 42-61. doi:10.1111/j.1749-6632.2010.05445.x

Amodio, D. M. (2019). Social Cognition 2.0: An Interactive Memory Systems Account. Trends in Cognitive Sciences, 23(1), 21-33. doi:https://doi.org/10.1016/j.tics.2018.10.002

Amodio, D. M., \& Frith, C. D. (2006). Meeting of minds: the medial frontal cortex and social cognition. Nat Rev Neurosci, 7(4), 268-277. Retrieved from http://dx.doi.org/10.1038/nrn1884

Badre, D. (2008). Cognitive control, hierarchy, and the rostro-caudal organization of the frontal lobes. Trends in Cognitive Sciences, 12(5), 193-200. Retrieved from http://www.sciencedirect.com/science/article/B6VH9-4S7S1C31/2/efdec53984418e739ae39c3781a8786f

Baldauf, D., \& Desimone, R. (2014). Neural Mechanisms of Object-Based Attention. Science, 344(6182), 424-427. doi:10.1126/science.1247003

Barrett, H. C. (2012). A hierarchical model of the evolution of human brain specializations. Proceedings of the National Academy of Sciences, 109(Supplement 1), 10733-10740. doi:10.1073/pnas.1201898109

Barrett, L. F., Adolphs, R., Marsella, S., Martinez, A. M., \& Pollak, S. D. (2019). Emotional Expressions Reconsidered: Challenges to Inferring Emotion From Human Facial Movements. Psychological Science in the Public Interest, 20(1), 1-68. doi:10.1177/1529100619832930

Belin, P., Zatorre, R. J., Lafaille, P., Ahad, P., \& Pike, B. (2000). Voice-selective areas in human auditory cortex. Nature, 403(6767), 309-312. doi:Doi 10.1038/35002078

Binney, R. J., \& Ramsey, R. (2019). Social Semantics: The role of conceptual knowledge and cognitive control in a neurobiological model of the social brain. PsyArXiv. doi:https://doi.org/10.31234/osf.io/36tm5

Bisley, J. W., \& Goldberg, M. E. (2003). Neuronal Activity in the Lateral Intraparietal Area and Spatial Attention. Science, 299(5603), 81-86. doi:10.1126/science.1077395

Bisley, J. W., \& Goldberg, M. E. (2010). Attention, Intention, and Priority in the Parietal Lobe. Annual Review of Neuroscience, 33(1), 1-21. doi:doi:10.1146/annurev-neuro-060909152823

Brass, M., Bekkering, H., Wohlschlager, A., \& Prinz, W. (2000). Compatibility between observed and executed finger movements: comparing symbolic, spatial, and imitative cues. Brain Cogn, 44(2), 124-143. Retrieved from http://www.ncbi.nlm.nih.gov/htbinpost/Entrez/query?db=m\&form=6\&dopt=r\&uid $=11041986$

Brass, M., Ruby, P., \& Spengler, S. (2009). Inhibition of imitative behaviour and social cognition. Philosophical Transactions of the Royal Society B: Biological Sciences, 364(1528), 2359-2367. doi:10.1098/rstb.2009.0066

Brooks, R. A. (1991). New Approaches to Robotics. Science, 253(5025), 1227-1232. doi:10.1126/science.253.5025.1227 
Bukach, C. M., Gauthier, I., \& Tarr, M. J. (2006). Beyond faces and modularity: the power of an expertise framework. Trends in Cognitive Sciences, 10(4), 159-166. doi:https://doi.org/10.1016/j.tics.2006.02.004

Bullmore, E., \& Sporns, O. (2009). Complex brain networks: graph theoretical analysis of structural and functional systems. Nat Rev Neurosci, 10(3), 186-198. doi:10.1038/nrn2575

Cabeza, R., \& Moscovitch, M. (2013). Memory Systems, Processing Modes, and Components: Functional Neuroimaging Evidence. Perspectives on Psychological Science, 8(1), 49-55. doi:10.1177/1745691612469033

Cacioppo, J. T., \& Berntson, G. G. (1992). Social Psychological Contributions to the Decade of the Brain - Doctrine of Multilevel Analysis. American Psychologist, 47(8), 1019-1028. doi:Doi 10.1037/0003-066x.47.8.1019

Caspers, S., Zilles, K., Laird, A. R., \& Eickhoff, S. B. (2010). ALE meta-analysis of action observation and imitation in the human brain. Neurolmage, 50(3), 1148-1167. Retrieved from http://www.sciencedirect.com/science/article/B6WNP-4Y34K4VF/2/abb15a27cda3449f47e0bf2cdbc932e8

Churchland, P. M. (2013). Matter and consciousness (3rd Edition ed.). Cambridge, MA: MIT Press.

Cisek, P. (2007). Cortical mechanisms of action selection: the affordance competition hypothesis. Philosophical Transactions of the Royal Society B: Biological Sciences, 362(1485), 1585-1599. doi:10.1098/rstb.2007.2054

Cisek, P., \& Kalaska, J. F. (2010). Neural Mechanisms for Interacting with a World Full of Action Choices. Annual Review of Neuroscience, 33(1), 269-298. doi:doi:10.1146/annurev.neuro.051508.135409

Cole, M. W., Reynolds, J. R., Power, J. D., Repovs, G., Anticevic, A., \& Braver, T. S. (2013). Multi-task connectivity reveals flexible hubs for adaptive task control. Nature Neuroscience, 16, 1348. doi:10.1038/nn.3470

https://www.nature.com/articles/nn.3470\#supplementary-information

Corbetta, M., Patel, G., \& Shulman, G. L. (2008). The Reorienting System of the Human Brain: From Environment to Theory of Mind. Neuron, 58(3), 306-324. Retrieved from http://www.sciencedirect.com/science/article/B6WSS-4SFRCHN5/2/6687475be4ba4737a0291df532dbb03a

Darda, K. M., Butler, E. E., \& Ramsey, R. (2018). Functional Specificity and Sex Differences in the Neural Circuits Supporting the Inhibition of Automatic Imitation. Journal of Cognitive Neuroscience, 30(6), 914-933. doi:10.1162/jocn_a_01261

Darda, K. M., \& Ramsey, R. (2019). The inhibition of automatic imitation: A meta-analysis and synthesis of fMRI studies. Neurolmage, 197, 320-329. doi:https://doi.org/10.1016/j.neuroimage.2019.04.059

Decety, J., \& Sommerville, J. A. (2003). Shared representations between self and other: a social cognitive neuroscience view. Trends Cogn Sci, 7(12), 527-533. Retrieved from http://www.ncbi.nlm.nih.gov/entrez/query.fcgi?cmd=Retrieve\&db=PubMed\&dopt= Citation\&list uids $=14643368$

Desimone, R., \& Duncan, J. (1995). Neural mechanisms of selective visual attention. Annual Review of Neuroscience, 18, 193-222. doi:10.1146/annurev.ne.18.030195.001205

Downing, P. E., Jiang, Y., Shuman, M., \& Kanwisher, N. (2001). A cortical area selective for visual processing of the human body. Science, 293(5539), 2470-2473. Retrieved from 
http://www.ncbi.nlm.nih.gov/entrez/query.fcgi?cmd=Retrieve\&db=PubMed\&dopt= Citation\&list uids $=11577239$

Downing, P. E., \& Peelen, M. V. (2011). The role of occipitotemporal body-selective regions in person perception. Cognitive Neuroscience, 1-24. doi:10.1080/17588928.2011.582945

Duncan, J. (2010). The multiple-demand (MD) system of the primate brain: mental programs for intelligent behaviour. Trends Cogn Sci, 14(4), 172-179. doi:10.1016/j.tics.2010.01.004

Duncan, J., Humphreys, G., \& Ward, R. (1997). Competitive brain activity in visual attention. Current Opinion in Neurobiology, 7(2), 255-261. doi:Doi 10.1016/S09594388(97)80014-1

Fecteau, J. H., \& Munoz, D. P. (2006). Salience, relevance, and firing: a priority map for target selection. Trends in Cognitive Sciences, 10(8), 382-390. doi:10.1016/j.tics.2006.06.011

Fedorenko, E., Duncan, J., \& Kanwisher, N. (2013). Broad domain generality in focal regions of frontal and parietal cortex. Proceedings of the National Academy of Sciences, 110(41), 16616-16621. doi:10.1073/pnas.1315235110

Fodor, J. A. (1983). The Modularity of Mind. Cambridge, MA: The MIT Press.

Frith, C. D., \& Frith, U. (1999). Interacting minds--a biological basis. Science, 286(5445), 1692-1695. Retrieved from http://www.ncbi.nlm.nih.gov/htbinpost/Entrez/query?db=m\&form=6\&dopt=r\&uid=10576727

Frith, C. D., \& Frith, U. (2012). Mechanisms of Social Cognition. Annual Review of Psychology, 63(1), 287-313. doi:doi:10.1146/annurev-psych-120710-100449

Frith, U., \& Frith, C., D. (2010). The social brain: allowing humans to boldly go where no other species has been. Philosophical Transactions of the Royal Society B: Biological Sciences, 365(1537), 165-176. doi:10.1098/rstb.2009.0160

Gazzola, V., Spezio, M. L., Etzel, J. A., Castelli, F., Adolphs, R., \& Keysers, C. (2012). Primary somatosensory cortex discriminates affective significance in social touch. Proceedings of the National Academy of Sciences, 109(25), E1657,ÄiE1666. doi:10.1073/pnas.1113211109

Georgieff, N., \& Jeannerod, M. (1998). Beyond Consciousness of External Reality: A "Who" System for Consciousness of Action and Self-Consciousness. Consciousness and Cognition, 7(3), 465-477. Retrieved from http://www.sciencedirect.com/science/article/B6WD0-45JK493K/2/ff918907b1d17af4f987734d2a75be22

Gerson, S. A., Bekkering, H., \& Hunnius, S. (2015). Short-term Motor Training, but Not Observational Training, Alters Neurocognitive Mechanisms of Action Processing in Infancy. Journal of Cognitive Neuroscience, 27(6), 1207-1214. doi:10.1162/jocn_a_00774\%M 25514654

Gray, K. (2017). How to Map Theory: Reliable Methods Are Fruitless Without Rigorous Theory. Perspect Psychol Sci, 12(5), 731-741. doi:10.1177/1745691617691949

Greven, I. M., Downing, P. E., \& Ramsey, R. (2016). Linking person perception and person knowledge in the human brain. Social Cognitive and Affective Neuroscience, 11(4), 641-651. doi:10.1093/scan/nsv148

Greven, I. M., \& Ramsey, R. (2017a). Neural network integration during the perception of ingroup and out-group members. Neuropsychologia, 106, 225-235. 
Greven, I. M., \& Ramsey, R. (2017b). Person perception involves functional integration between the extrastriate body area and temporal pole. Neuropsychologia, 96, 52-60. doi:http://dx.doi.org/10.1016/j.neuropsychologia.2017.01.003

Grossman, E., Donnelly, M., Price, R., Pickens, D., Morgan, V., Neighbor, G., \& Blake, R. (2000). Brain Areas Involved in Perception of Biological Motion. Journal of Cognitive Neuroscience, 12(5), 711-720. doi:doi:10.1162/089892900562417

Insel, T. R. (2003). Is social attachment an addictive disorder? Physiology \& Behavior, 79(3), 351-357. doi:https://doi.org/10.1016/S0031-9384(03)00148-3

Insel, T. R., \& Fernald, R. D. (2004). HOW THE BRAIN PROCESSES SOCIAL INFORMATION: Searching for the Social Brain. Annual Review of Neuroscience, 27(1), 697-722. doi:10.1146/annurev.neuro.27.070203.144148

Jefferies, E. (2013). The neural basis of semantic cognition: Converging evidence from neuropsychology, neuroimaging and TMS. Cortex, 49(3), 611-625. doi:10.1016/j.cortex.2012.10.008

Kanwisher, N. (2010). Functional specificity in the human brain: A window into the functional architecture of the mind. Proceedings of the National Academy of Sciences, 107(25), 11163-11170. doi:10.1073/pnas.1005062107

Kanwisher, N. (2017). The Quest for the FFA and Where It Led. The Journal of Neuroscience, 37(5), 1056-1061. doi:10.1523/jneurosci.1706-16.2016

Kanwisher, N., McDermott, J., \& Chun, M. M. (1997). The Fusiform Face Area: A Module in Human Extrastriate Cortex Specialized for Face Perception. J. Neurosci., 17(11), 4302-4311. Retrieved from http://www.jneurosci.org/cgi/content/abstract/17/11/4302

Kelley, A. E., \& Berridge, K. C. (2002). The Neuroscience of Natural Rewards: Relevance to Addictive Drugs. The Journal of Neuroscience, 22(9), 3306-3311. doi:10.1523/jneurosci.22-09-03306.2002

Krach, S., Paulus, F., Bodden, M., \& Kircher, T. (2010). The rewarding nature of social interactions. Frontiers in Behavioral Neuroscience, 4(22). doi:10.3389/fnbeh.2010.00022

Krall, S. C., Rottschy, C., Oberwelland, E., Bzdok, D., Fox, P. T., Eickhoff, S. B., . . Konrad, K. (2015). The role of the right temporoparietal junction in attention and social interaction as revealed by ALE meta-analysis. Brain Structure and Function, 220(2), 587-604. doi:10.1007/s00429-014-0803-z

Kuzmanovic, B., Jefferson, A., Bente, G., \& Vogeley, K. (2013). Affective and motivational influences in person perception. Frontiers in Human Neuroscience, $7(266)$. doi:10.3389/fnhum.2013.00266

Lambon Ralph, M. A., Jefferies, E., Patterson, K., \& Rogers, T. T. (2017). The neural and computational bases of semantic cognition. Nature Reviews Neuroscience, 18, 42-55. doi:10.1038/nrn.2016.150

https://www.nature.com/articles/nrn.2016.150\#supplementary-information

Leslie, A. M., German, T. P., \& Polizzi, P. (2005). Belief-desire reasoning as a process of selection. Cognit Psychol, 50(1), 45-85. Retrieved from http://www.ncbi.nlm.nih.gov/entrez/query.fcgi?cmd=Retrieve\&db=PubMed\&dopt= Citation\&list uids $=15556129$

Leslie, A. M., \& Thaiss, L. (1992). Domain specificity in conceptual development: Neuropsychological evidence from autism. Cognition, 43(3), 225-251. doi:10.1016/0010-0277(92)90013-8 
Lieberman, M. D. (2007). Social cognitive neuroscience: A review of core processes. Annual Review of Psychology, 58, 259-289. doi:10.1146/annurev.psych.58.110405.085654

Loken, L. S., Wessberg, J., Morrison, I., McGlone, F., \& Olausson, H. (2009). Coding of pleasant touch by unmyelinated afferents in humans. Nature Neuroscience, 12(5), 547-548. doi:10.1038/nn.2312

Meyer, M., Bekkering, H., Haartsen, R., Stapel, J. C., \& Hunnius, S. (2015). The role of action prediction and inhibitory control for joint action coordination in toddlers. Journal of Experimental Child Psychology, 139, 203-220. doi:https://doi.org/10.1016/j.jecp.2015.06.005

Michael, J., \& D’Ausilio, A. (2015). Domain-specific and domain-general processes in social perception - A complementary approach. Consciousness and Cognition, 36(Supplement C), 434-437. doi:https://doi.org/10.1016/i.concog.2014.12.009

Miller, E. K. (2000). The prefrontal cortex and cognitive control. Nat Rev Neurosci, 1(1), 5965. Retrieved from http://www.ncbi.nlm.nih.gov/htbinpost/Entrez/query?db=m\&form=6\&dopt=r\&uid=11252769

Mitchell, J. P., Cloutier, J., Banaji, M. R., \& Macrae, C. N. (2006). Medial prefrontal dissociations during processing of trait diagnostic and nondiagnostic person information. Social Cognitive and Affective Neuroscience, 1(1), 49-55. doi:10.1093/scan/nsl007

Mitchell, J. P., Heatherton, T. F., \& Macrae, C. N. (2002). Distinct neural systems subserve person and object knowledge. Proc. Natl. Acad. Sci. USA, 99, 15238.

Molenberghs, P., Cunnington, R., \& Mattingley, J. B. (2012). Brain regions with mirror properties: A meta-analysis of 125 human fMRI studies. Neuroscience \&amp; Biobehavioral Reviews, 36(1), 341-349. doi:10.1016/j.neubiorev.2011.07.004

Morrison, I., Löken, L. S., \& Olausson, H. (2010). The skin as a social organ. Experimental Brain Research, 204(3), 305-314. doi:10.1007/s00221-009-2007-y

Munafò, M. R., Nosek, B. A., Bishop, D. V. M., Button, K. S., Chambers, C. D., Percie du Sert, N., . . Ioannidis, J. P. A. (2017). A manifesto for reproducible science. Nature Human Behaviour, 1, 0021. doi:10.1038/s41562-016-0021

Over, H., \& Cook, R. (2018). Where do spontaneous first impressions of faces come from? Cognition, 170, 190-200. doi:10.1016/j.cognition.2017.10.002

Park, H.-J., \& Friston, K. (2013). Structural and Functional Brain Networks: From Connections to Cognition. Science, 342(6158). Retrieved from http://science.sciencemag.org/content/342/6158/1238411.abstract

Patel, G. H., Sestieri, C., \& Corbetta, M. (2019). The evolution of the temporoparietal junction and posterior superior temporal sulcus. Cortex, 118, 38-50. doi:https://doi.org/10.1016/j.cortex.2019.01.026

Pessoa, L., \& Adolphs, R. (2010). Emotion processing and the amygdala: from a 'low road' to 'many roads' of evaluating biological significance. Nature Reviews Neuroscience, 11(11), 773-782. doi:10.1038/nrn2920

Petersen, S. E., \& Posner, M. I. (2012). The Attention System of the Human Brain: 20 Years After. Annual Review of Neuroscience, Vol 35, 35, 73-89. doi:10.1146/annurevneuro-062111-150525

Poldrack, R. A. (2006). Can cognitive processes be inferred from neuroimaging data? Trends Cogn Sci, 10(2), 59-63. Retrieved from http://www.ncbi.nlm.nih.gov/entrez/query.fcgi?cmd=Retrieve\&db=PubMed\&dopt= Citation\&list uids $=16406760$ 
Ptak, R. (2012). The Frontoparietal Attention Network of the Human Brain:Action, Saliency, and a Priority Map of the Environment. The Neuroscientist, 18(5), 502-515. doi:10.1177/1073858411409051

Puce, A., \& Perrett, D. (2003). Electrophysiology and brain imaging of biological motion. Philos Trans R Soc Lond B Biol Sci, 358(1431), 435-445. Retrieved from http://www.ncbi.nlm.nih.gov/entrez/query.fcgi?cmd=Retrieve\&db=PubMed\&dopt= Citation\&list uids $=12689371$

Quadflieg, S., Flannigan, N., Waiter, G. D., Rossion, B., Wig, G. S., Turk, D. J., \& Macrae, C. N. (2011). Stereotype-based modulation of person perception. Neurolmage, 57(2), 549557. doi:10.1016/j.neuroimage.2011.05.004

Ramsey, R. (2018a). Neural Integration in Body Perception. Journal of Cognitive Neuroscience, 30(10), 1442-1451. doi:10.1162/jocn_a_01299

Ramsey, R. (2018b). What are reaction time indices of automatic imitation measuring? Consciousness and Cognition, 65, 240-254. doi:https://doi.org/10.1016/j.concog.2018.08.006

Ramsey, R., Hansen, P., Apperly, I., \& Samson, D. (2013). Seeing It My Way or Your Way: Frontoparietal Brain Areas Sustain Viewpoint-independent Perspective Selection Processes. Journal of Cognitive Neuroscience, 25(5), 670-684. doi:10.1162/jocn_a_00345

Rushworth, M. F. S., Buckley, M. J., Behrens, T. E. J., Walton, M. E., \& Bannerman, D. M. (2007). Functional organization of the medial frontal cortex. Current Opinion in Neurobiology, 17(2), 220-227. doi:https://doi.org/10.1016/i.conb.2007.03.001

Saxe, R., \& Kanwisher, N. (2003). People thinking about thinking people. The role of the temporo-parietal junction in "theory of mind". Neurolmage, 19(4), 1835-1842. Retrieved from http://www.ncbi.nlm.nih.gov/entrez/query.fcgi?cmd=Retrieve\&db=PubMed\&dopt= Citation\&list uids $=12948738$

Scholz, J., Triantafyllou, C., Whitfield-Gabrieli, S., Brown, E. N., \& Saxe, R. (2009). Distinct Regions of Right Temporo-Parietal Junction Are Selective for Theory of Mind and Exogenous Attention. PLOS ONE, 4(3), e4869. Retrieved from http://dx.doi.org/10.1371\%2Fjournal.pone.0004869

Serences, J. T., \& Yantis, S. (2006). Selective visual attention and perceptual coherence. Trends in Cognitive Sciences, 10(1), 38-45. doi:10.1016/j.tics.2005.11.008

Shallice, T., \& Cipolotti, L. (2018). The Prefrontal Cortex and Neurological Impairments of Active Thought. Annual Review of Psychology, 69(1), 157-180. doi:10.1146/annurevpsych-010416-044123

Simons, D. J., Shoda, Y., \& Lindsay, D. S. (2017). Constraints on Generality (COG): A Proposed Addition to All Empirical Papers. Perspectives on Psychological Science, 12(6), 11231128. doi:10.1177/1745691617708630

Spengler, S., von Cramon, D. Y., \& Brass, M. (2009). Control of shared representations relies on key processes involved in mental state attribution. Human brain mapping, 30(11), 3704-3718.

Sporns, O. (2013). Network attributes for segregation and integration in the human brain. Curr Opin Neurobiol, 23(2), 162-171. doi:10.1016/j.conb.2012.11.015

Spunt, R. P., \& Adolphs, R. (2017). A new look at domain specificity: insights from social neuroscience. Nat Rev Neurosci, 18(9), 559-567. doi:10.1038/nrn.2017.76 
Spunt, R. P., \& Adolphs, R. (2019). The neuroscience of understanding the emotions of others. Neuroscience Letters, 693, 44-48. doi:https://doi.org/10.1016/j.neulet.2017.06.018

Tarr, M. J., \& Gauthier, I. (2000). FFA: a flexible fusiform area for subordinate-level visual processing automatized by expertise. Nature Neuroscience, 3(8), 764-769. doi:10.1038/77666

van Elk, M., van Schie, H., \& Bekkering, H. (2014). Action semantics: A unifying conceptual framework for the selective use of multimodal and modality-specific object knowledge. Physics of Life Reviews, 11(2), 220-250. doi:https://doi.org/10.1016/j.plrev.2013.11.005

Van Overwalle, F. (2009). Social cognition and the brain: a meta-analysis. Hum. Brain Mapp., $30,829$.

Vuilleumier, P., \& Pourtois, G. (2007). Distributed and interactive brain mechanisms during emotion face perception: Evidence from functional neuroimaging. Neuropsychologia, 45(1), 174-194. doi:http://dx.doi.org/10.1016/j.neuropsychologia.2006.06.003

Wang, Y., Collins, J. A., Koski, J., Nugiel, T., Metoki, A., \& Olson, I. R. (2017). Dynamic neural architecture for social knowledge retrieval. Proceedings of the National Academy of Sciences, 114(16), E3305-E3314.

Wang, Y., \& Hamilton, A. F. d. C. (2012). Social Top-down Response Modulation (STORM): A model of the control of mimicry in social interaction. Frontiers in Human Neuroscience, 6. doi:10.3389/fnhum.2012.00153

Wang, Y., Ramsey, R., \& Hamilton, A. F. d. C. (2011). The Control of Mimicry by Eye Contact Is Mediated by Medial Prefrontal Cortex. The Journal of Neuroscience, 31(33), 1200112010. doi:10.1523/jneurosci.0845-11.2011

Ward, R. (1999). Interaction between perception and action systems: a model for selective action. In Humphreys, G.W., Duncan, J. and Treisman, A. (Eds.) Attention, Space and Action: Studies in Cognitive Neuroscience (pp. 311-332). Oxford University Press, New York.

Weigelt, S., Koldewyn, K., Dilks, D. D., Balas, B., McKone, E., \& Kanwisher, N. (2014). Domain-specific development of face memory but not face perception. Developmental Science, 17(1), 47-58. doi:10.1111/desc.12089

Yovel, G., \& Kanwisher, N. (2004). Face Perception: Domain Specific, Not Process Specific. Neuron, 44(5), 889-898. doi:https://doi.org/10.1016/j.neuron.2004.11.018 